\title{
Correction to: Magnitude and direction of the association between Clostridium difficile infection and proton pump inhibitors in adults and pediatric patients: a systematic review and meta- analysis
}

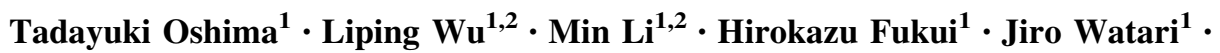
Hiroto Miwa ${ }^{1}$

Published online: 30 January 2020

(C) Japanese Society of Gastroenterology 2020

Correction to: J Gastroenterol (2018) 53:84-94

https://doi.org/10.1007/s00535-017-1369-3

In the original publication of the article, Table 1 and Figs. 2a, 2b, 3a were published with errors. The correct Table 1 and Figs. 2a, 2b, 3a are given in this correction.

The original article can be found online at https://doi.org/10.1007/ s00535-017-1369-3.

Tadayuki Oshima

t-oshima@hyo-med.ac.jp

1 Division of Gastroenterology, Department of Internal Medicine, Hyogo College of Medicine, 1-1 Mukogawa-cho,

Nishinomiya, Hyogo 663-8501, Japan

2 Department of Gastroenterology, The Third People's Hospital of Chengdu, Chengdu, China 
Table 1 Characteristics of the included studies

\begin{tabular}{|c|c|c|c|c|c|c|}
\hline Author & Year & Country & Study type & Setting & Recurrence or not & Duration of PPI use \\
\hline Shah et al. [40] & 2000 & UK & Case-control & Inpatient & New patient & $<16$ weeks \\
\hline Cunningham et al. [41] & 2003 & UK & Case-control & Inpatient & New patient & $<2$ months \\
\hline Dial et al. [42] & 2004 & Canada & Both & Inpatient & New patient & Not specified \\
\hline Dial et al. [43] & 2005 & UK & Case-control & Community & New patient & $<90$ day \\
\hline Modena et al. [44] & 2005 & USA & Case-control & Inpatient & New patient & Not specified \\
\hline Al-Tureihi et al. [45] & 2005 & USA & Case-control & Inpatient & New patient & Not specified \\
\hline Loo et al. [35] & 2005 & Canada & Case-control & Inpatient & New patient & $\leq 6$ weeks \\
\hline Muto et al. [46] & 2005 & USA & Case-control & Inpatient & New patient & Not specified \\
\hline Kazakova et al. [47] & 2006 & USA & Case-control & Inpatient & New patient & Not specified \\
\hline Yearsley et al. [36] & 2006 & UK & Case-control & Inpatient & New patient & $<90$ Day \\
\hline Lowe et al. [48] & 2006 & Canada & Case-control & Inpatient & New patient & Specified \\
\hline Dial et al. [49] & 2006 & UK & Case-control & Community & New patient & $<90$ Day \\
\hline Akhtar et al. [50] & 2007 & UK & Case-control & Inpatient & New patient & Not specified \\
\hline Jayatilaka et al. [51] & 2007 & USA & Case-control & Inpatient & New patient & Not specified \\
\hline Dubberke et al. [52] & 2007 & USA & Cohort & Inpatient & New patient & Not specified \\
\hline Cadle et al. [53] & 2007 & USA & Cohort & Inpatient & Recurrence & 7 days or more \\
\hline McFarland et al. [54] & 2007 & USA & Case-control & Inpatient & New patient & Not specified \\
\hline Aseeri et al. [55] & 2008 & USA & Case-control & Inpatient & New patient & Not specified \\
\hline Baxter et al. [56] & 2008 & USA & Case-control & Inpatient & New patient & Not specified \\
\hline Debast et al. [57] & 2009 & The Netherlands & Case-control & Inpatient & New patient & Not specified \\
\hline Dalton et al. [58] & 2009 & Canada & Cohort & Inpatient & New patient & $18.7 \pm 21.5$ \\
\hline Kim et al. [59] & 2010 & South Korea & Case-control & Inpatient & Recurrence & Not specified \\
\hline Howell et al. [37] & 2010 & USA & Cohort & Inpatient & New patient & Not specified \\
\hline Linsky et al. [60] & 2010 & USA & Cohort & Both & Recurrence & Not specified \\
\hline Turco et al. [61] & 2010 & Italy & Case-control & Inpatient & New patient & Not specified \\
\hline Bajaj et al. [62] & 2010 & USA & Case-control & Inpatient & New patient & Not specified \\
\hline Linney et al. [63] & 2010 & USA & Case-control & Inpatient & New patient & Not specified \\
\hline Manges et al. [64] & 2010 & Canada & Case-control & Inpatient & New patient & Not specified \\
\hline Jenkins et al. [65] & 2010 & UK & Case-control & Inpatient & New patient & Not specified \\
\hline Stevens et al. [66] & 2011 & USA & Cohort & Inpatient & New patient & Not specified \\
\hline Naggie et al. [67] & 2011 & USA & Case-control & Community & New patient & Not specified \\
\hline Hensgens et al. [68] & 2011 & The Netherlands & Case-control & Inpatient & New patient & Not specified \\
\hline Loo et al. [5] & 2011 & Canada & Cohort & Inpatient & New patient & $<8$ weeks \\
\hline Kim et al. [69] & 2012 & South Korea & Case-control & Inpatient & Recurrence & $<2$ months \\
\hline Leonard et al. [70] & 2012 & UK & Case-control & Inpatient & New patient & $>30$ days \\
\hline Vesteinsdottir et al. [38] & 2012 & Iceland & Case-control & Inpatient & New patient & Not specified \\
\hline Lupse et al. [71] & 2013 & Romania & Case-control & Inpatient & Recurrence & Not specified \\
\hline Samie et al. [72] & 2013 & Germany & Case-control & Inpatient & Recurrence & Not specified \\
\hline Mizui et al. [73] & 2013 & Japan & Case-control & Inpatient & New patient & Not specified \\
\hline Freedberg et al. [74] & 2013 & USA & Case-control & Inpatient & Recurrence & $<3$ months \\
\hline Campbell et al. [75] & 2013 & USA & Case-control & Inpatient & New patient & Not specified \\
\hline Barletta et al. [76] & 2013 & USA & Case-control & Inpatient & New patient & 5 days \\
\hline Rodríguez-Pardo et al. [7] & 2013 & Spain & Case-control & Inpatient & Recurrence & Not specified \\
\hline Hebert et al. [8] & 2013 & USA & Cohort & Inpatient & Recurrence & Not specified \\
\hline Wang et al. [39] & 2014 & China & Cohort & Inpatient & New patient & Not specified \\
\hline Pakyz et al. [77] & 2014 & USA & Case-control & Inpatient & New patient & Not specified \\
\hline Novack et al. [15] & 2014 & USA & Case-control & Inpatient & New patient & Not specified \\
\hline Barletta et al. [78] & 2014 & USA & Case-control & Inpatient & New patient & Not specified \\
\hline
\end{tabular}


Table 1 continued

\begin{tabular}{|c|c|c|c|c|c|c|}
\hline Author & Year & Country & Study type & Setting & Recurrence or not & Duration of PPI use \\
\hline Samady et al. [79] & 2014 & USA & Case-control & Inpatient & New patient & Not specified \\
\hline Zhu et al. [80] & 2014 & China & Case-control & Inpatient & New patient & Not specified \\
\hline Sathyendran et al. [81] & 2014 & New Zealand & Case-control & Inpatient & New patient & Not specified \\
\hline Brown et al. [16] & 2015 & USA & Case-control & Inpatient & New patient & Not specified \\
\hline Freedberg et al. [82] & 2015 & USA & Case-control & Inpatient & New patient & Not specified \\
\hline Abdelfatah et al. [83] & 2015 & USA & Case-control & Both & Recurrence & Not specified \\
\hline McDonald et al. [84] & 2015 & Canada & Cohort & Inpatient & Recurrence & $<3$ months \\
\hline Jimenez et al. [85] & 2015 & USA & Case-control & Both & New patient & Not specified \\
\hline Ticinesi et al. [86] & 2015 & Italy & Cohort & Inpatient & New patient & Not specified \\
\hline Hikone et al. [87] & 2015 & Japan & Cohort & Both & Recurrence & Not specified \\
\hline Ramos-Martínez et al. [88] & 2016 & Spain & Case-control & Inpatient & New patient & Not specified \\
\hline Depoorter et al. [89] & 2016 & Belgium & Case-control & Inpatient & New patient & Not specified \\
\hline Faleck et al. [22] & 2016 & USA & Cohort & Inpatient & New patient & Not specified \\
\hline Gordon et al. [90] & 2016 & USA & Cohort & Both & New patient & Not specified \\
\hline Imlay et al. [91] & 2016 & USA & Case-control & Both & New patient & Not specified \\
\hline Lewis et al. [92] & 2016 & USA & Cohort & Both & New patient & Not specified \\
\hline Ramos et al. [93] & 2016 & Spain & Case-control & Inpatient & New patient & $<3$ months \\
\hline Ro et al. [94] & 2016 & South Korea & Cohort & Inpatient & New patient & Not specified \\
\hline Rogala et al. [95] & 2016 & USA & Case-control & Inpatient & New patient & $<3$ months \\
\hline
\end{tabular}


Figure 2

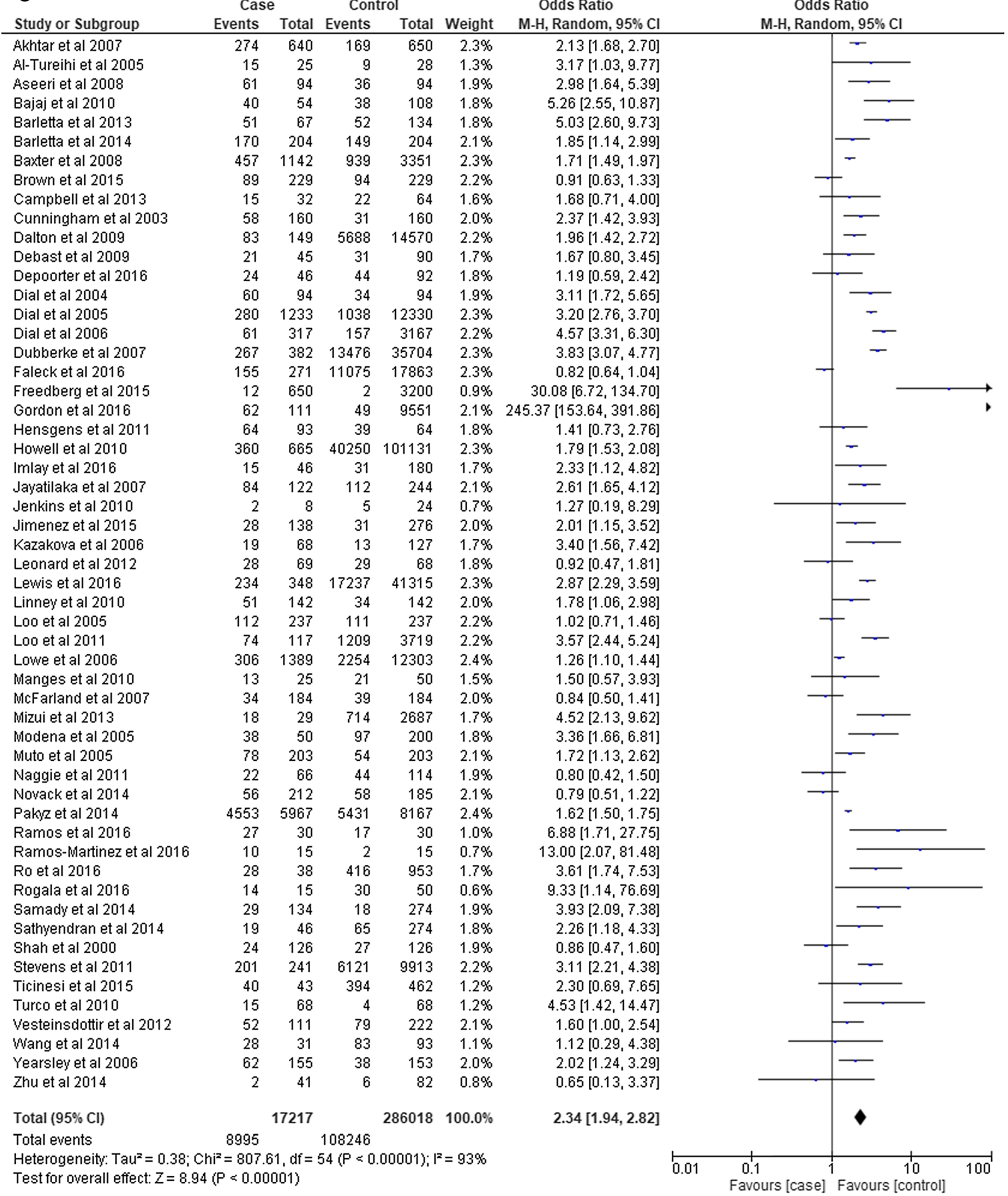




\section{Figure 2}

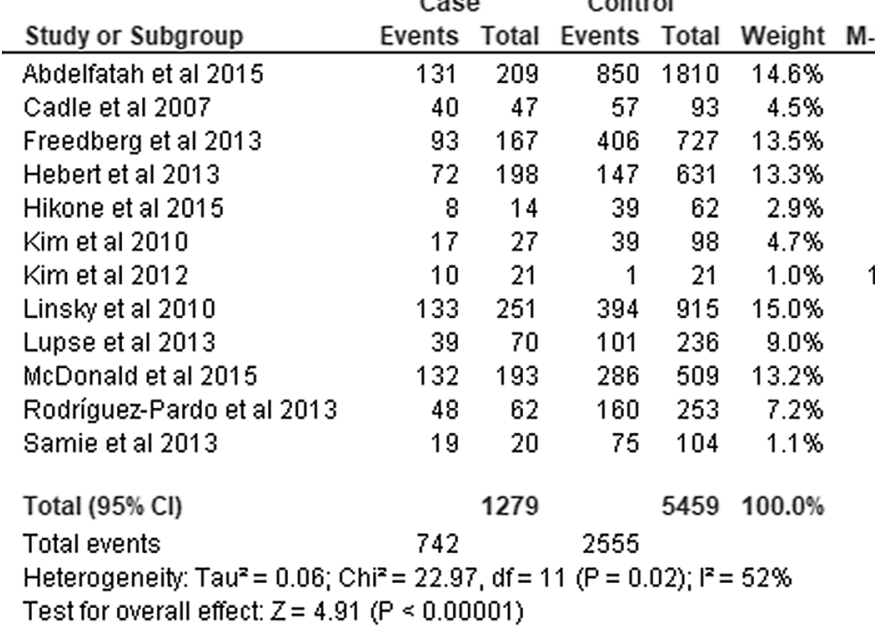

Odds Ratio

H, Random, $95 \% \mathrm{Cl}$

$1.90[1.41,2.55]$

$3.61[1.46,8.92]$

$0.99[0.71,1.39]$

$1.88[1.33,2.65]$

$0.79[0.24,2.55]$

$2.57[1.07,6.20]$

$18.18[2.05,161.38]$

$1.49[1.13,1.97]$

$1.68[0.98,2.88]$

$1.69[1.19,2.40]$

$1.99[1.04,3.81]$

$7.35[0.94,57.41]$

$1.73[1.39,2.15]$

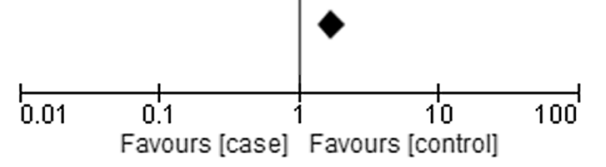


Figure 3

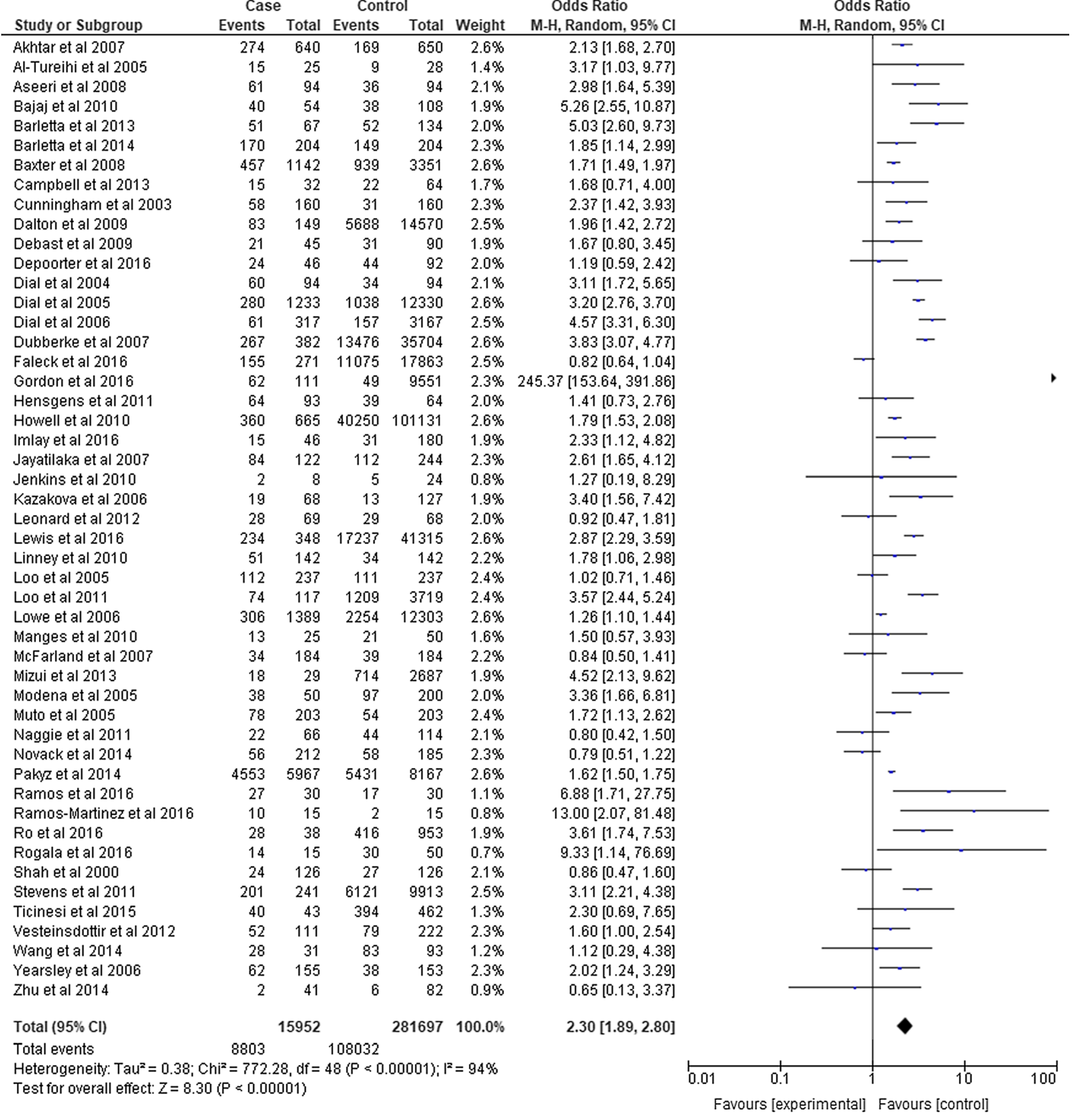

